\title{
Computer-aided detection of fasciculations and other movements in muscle with ultrasound: Development and clinical application
}

\author{
Kaj Gijsbertse ${ }^{\mathrm{a}, 1}$, Max Bakker ${ }^{\mathrm{a}, *, 1}$, André Sprengers ${ }^{\mathrm{a}, \mathrm{b}}$, Juerd Wijntjes ${ }^{\mathrm{c}}$, Saskia Lassche ${ }^{\mathrm{c}}$, \\ Nico Verdonschot ${ }^{\mathrm{a}, \mathrm{b}}$, Chris L. de Korte ${ }^{\mathrm{d}, \mathrm{e}}$, Nens van Alfen ${ }^{\mathrm{c}}$ \\ a Orthopaedic Research Laboratory, Department of Orthopaedics, Radboud University Medical Center, Nijmegen, The Netherlands \\ ${ }^{\mathrm{b}}$ Laboratory of Biomechanical Engineering, University of Twente, Enschede, The Netherlands \\ ${ }^{\mathrm{c}}$ Department of Neurology and Clinical Neurophysiology, Donders Institute for Brain, Cognition and Behaviour, Radboud University Medical Center, Nijmegen, The Netherlands \\ ${ }^{\mathrm{d}}$ Medical Ultrasound Imaging Center (MUSIC), Department of Radiology and Nuclear Medicine, Radboud University Medical Center, Nijmegen, The Netherlands

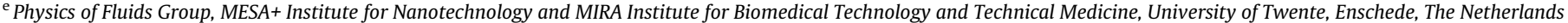

\section{A R T I C L E I N F O}

Article history:

Accepted 30 September 2018

Available online 27 October 2018

Keywords:

Ultrasound

Fasciculations

Computer-aided detection

ALS

\section{H I G H L I G H T S}

- We developed an automatic algorithm for detecting fasciculations in muscle ultrasound videos.

- With algorithm guidance, observers found more fasciculations compared to visual analysis alone.

- Our findings affirm the potential clinical usefulness of automated analysis of muscle ultrasound.

\begin{abstract}
A B S T R A C T
Objective: To develop an automated algorithm for detecting fasciculations and other movements in muscle ultrasound videos. Fasciculation detection in muscle ultrasound is routinely performed online by observing the live videos. However, human observation limits the objective information gained. Automated detection of movement is expected to improved sensitivity and specificity and increase reliability. Methods: We used 42 ultrasound videos from 11 neuromuscular patients for an iterative learning process between human observers and automated computer analysis, to identify muscle ultrasound movements. Two different datasets were selected from this, one to develop the algorithm and one to validate it. The outcome was compared to manual movement identification by clinicians. The algorithm also quantifies specific parameters of different movement types, to enable automated differentiation of events.

Results: The algorithm reliably detected fasciculations. With algorithm guidance, observers found more fasciculations compared to visual analysis alone, and prescreening the videos with the algorithm saved clinicians significant time compared to reviewing full video sequences. All videos also contained other movements, especially contraction pseudotremor, which confused human interpretation in some.

Conclusions: Automated movement detection is a feasible and attractive method to screen for fasciculations in muscle ultrasound videos.

Significance: Our findings affirm the potential clinical usefulness of automated movement analysis in muscle ultrasound.
\end{abstract}

(c) 2018 International Federation of Clinical Neurophysiology. Published by Elsevier B.V. All rights reserved.

\section{Introduction}

Muscle ultrasound imaging is an increasingly important addition to the diagnostic arsenal for diagnosing neuromuscular dis-

\footnotetext{
* Corresponding author.

E-mail address: Max.Bakker@radboudumc.nl (M. Bakker).

1 Authors contributed equally; K Gijsbertse and M Bakker share first authorship of this article.
}

ease, providing an anatomical assessment of muscle structure to complement standard neurological examination and electrophysiologic function testing (Simon, 2015). In addition to its well-known advantages of being patient-friendly, non-invasive and a point of care imaging technique, the dynamic nature of ultrasound images as a result of the high temporal resolution enables visualization of spontaneous or voluntary muscle movements, including fasciculations. 
Fasciculations are spontaneous contractions of a group of muscle fibers innervated by a single motor axon (i.e., one motor unit). They can be physiologic in certain muscles under specific circumstances (such as calf fasciculations after sports activities), but in the context of clinical symptoms they are often a sign of motor neuron or peripheral nerve pathology. Since the Awaji modification of the El Escorial criteria for amyotrophic lateral sclerosis (ALS), fasciculations are an important clue to confirm the involvement of the peripheral nervous system in patients with suspected ALS (Geevasinga et al., 2016). In ALS, ultrasound has a consistently higher detection rate of fasciculations compared to clinical examination (e.g., visual inspection) or electromyography (EMG) (Grimm et al., 2015, Misawa et al., 2011, Regensburger et al., 2017).

However, visual detection of such brief muscle contractions within ultrasound image sequences by a human observer is time consuming and subjective, resulting in under-detection and relevant intra- and inter observer variation (Pillen et al., 2009, Reimers et al., 1996). To overcome these limitations, computeraided techniques have been introduced for automated image interpretation. Only one previous study has reported on the application of computer-aided techniques in the detection of fasciculations (Harding et al., 2016). The authors proposed an optical flow technique to quantify muscle motion and calculate mutual information, to measure the interdependence of the motion and discriminate between muscle twitches (e.g., fasciculations) and muscle tissue at rest. They found good agreement between manual and computational detection of muscle twitches in two different muscles (biceps brachii and medial gastrocnemius). However, not all twitches in muscle tissue observed during ultrasound are fasciculations. Other tissue movements caused by voluntary contractions of small parts of a muscle (including contraction pseudotremor, which is the isolated contraction of enlarged, neurogenic motor units during slight antigravity movement), muscle fibrillation after denervation, vascular pulsations, and imaging artefacts such as probe motion or scatter artefacts close to bony surfaces, can appear on the ultrasound recordings. For the purpose of helping the clinician to detect and quantify fasciculations, automated ultrasound analysis should not only detect, but also discriminate fasciculations from these other types of muscle ultrasound movements. To do this, physiologic information on the specific characteristics of these different types of motion is required to distinguish between the different observed movements in the ultrasound data.

Using a human observer as the gold standard for detection of a specific type of movement in an ultrasound video is potentially prone to error, especially when many simultaneous movements are present in the real-time image flow, as human observation and interpretation will select some features for its attention but will ignore others. To overcome this, observers would need to selectively look at every image region (or pixel, ideally) in a frame-by-frame approach, which is expected to be more accurate, but is also very time consuming and unsuitable for routine clinical evaluation of an ultrasound study. However, initially such a frame-by-frame evaluation is necessary in a certain image set, to develop/train an automated detection algorithm.

In this study we introduce a computationally cheap framework for the automatic detection of motion within ultrasound images sequences. We take the first steps of an iterative process that starts with human observation, then compare the results to automated detection of any image motion using an ultrasound background subtraction-based method, feed this information back to the human observer who next selectively evaluates every movement detected in a frame-by-frame visual analysis. With this approach, we provide physiologic information of the detected motions that might be used to classify the different detected events. In this paper, we show that this approach can detect fasciculations (i.e., has sensitivity), and is potentially able to differentiate fasciculations from other movements (i.e., has specificity), paving the way towards automatic classification using machine learning.

\section{Methods}

\section{A. Clinical and ultrasound data collection}

This retrospective study retrieved a set of 42 ultrasound image sequences (i.e., videos) from 11 patients seen at the neuromuscular outpatient clinic of the Radboud university medical center (Fig. 1, step A). All procedures followed were in accordance with the ethical standards of the responsible committee on human experimentation and with the Helsinki Declaration of 1975. As per a general rule of the Dutch ethics committee, the studied needed no further ethical approval procedure, because the study protocol only involved the post-hoc review of anonymized data that had been captured during routine clinical care. Patients had been referred for the workup of different neuromuscular disorders, such as suspected myopathy, unexplained myalgia and fatigue, unexplained extremity or axial weakness, suspected motor neuron disease, and hereditary polyneuropathy. The ultrasound videos were retrieved from different muscles. Patient characteristics and muscles studied can be found in Table 1. All ultrasound examinations were performed on an Esaote MyLab Twice system (Esaote, Genoa - Italy), equipped with a 3-13 MHz broadband linear transducer (LA533), using a standard pre-set and image parameters described earlier (Scholten et al., 2003). Image depth was set at $4 \mathrm{~cm}$ for all muscles except the rectus femoris, for which the depth was set at $6 \mathrm{~cm}$. For every acquisition, 30 seconds of ultrasound video were recorded from the relaxed muscle, using a framerate of $20 \mathrm{~Hz}$. All ultrasound data were initially scored by experienced clinicians (JW, NvA, SL); see below Fig. 1, step B. To develop the algorithm (section D), a training set of 5 videos was selected from the available videos by the main researcher (KG; Fig. 1, step C.1; also see Table 2) to include a variation of image sequences that contained few, some or many movement events. Subsequently, another subset consisting of 5 videos was selected to test the developed algorithm i.e., test-set (Fig. 1, step E.2; also see Table 3). More details of the selection of the data-sets are provided in their corresponding section; see below section $C$ and section $\mathrm{E}$.

\section{B. Observer study}

For the initial scoring of the videos (see Fig. 1, step B), the ultrasound image sequences were visually assessed by three experienced neuromuscular clinicians: 2 clinical neurophysiologists (NvA, JW) and one neuromuscular fellow (SL). The clinicians were asked to state whether fasciculations were present or not in the image sequences, and to count the total number of fasciculations (NF) during the $30 \mathrm{~s}$ video. Any other present movement, such as vascular pulsation, probe motion artefacts or voluntary muscle contraction, were scored by the observers as well. Muscle echogenicity was assessed from the ultrasound videos, and scored semi-quantitatively by using the Heckmatt grading scale (Heckmatt et al., 1982). This scale represents a visual grading of muscle echogenicity which corresponds to changes in muscle tissue architecture such as fibrosis and fatty degeneration, with the rating scale as follows: 1-normal, 2-mildy increased muscle echoes with normal bone reflection, 3- moderately increased muscle echoes with reduced bone reflection, 4-severely increased muscle echoes with absent bone reflection. 


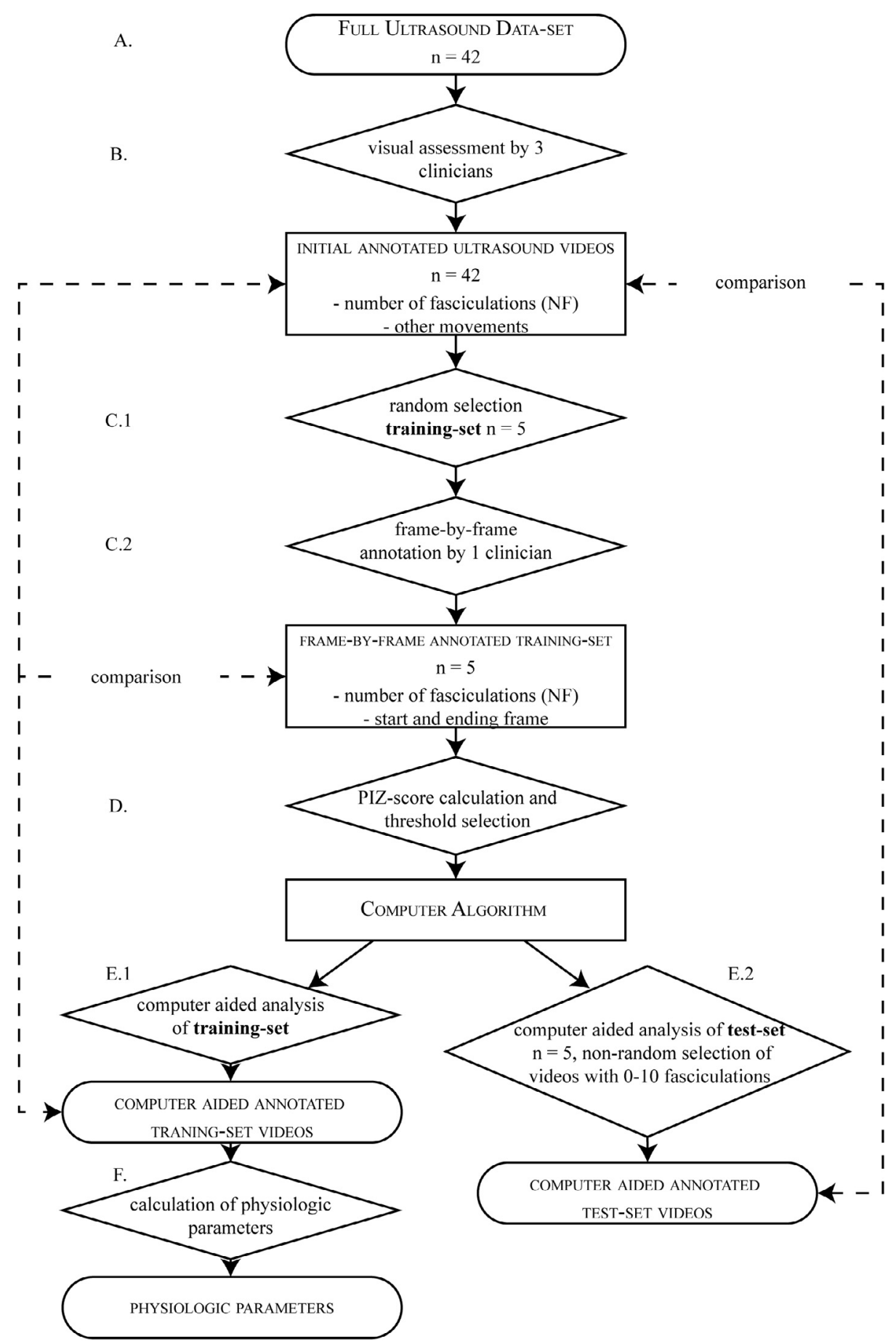

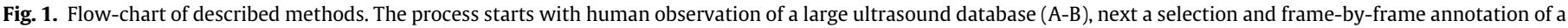

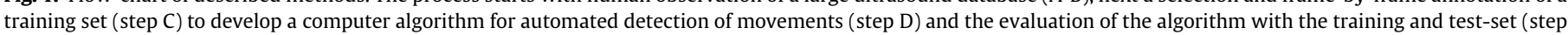
E.1 and E.2). Finally, physiologic parameters for the different muscles were extracted from the training set (step F).

\section{C. Training set for algorithm development}

To develop the algorithm, a subset of 5 ultrasound image sequences ( $30 \mathrm{~s}$ epochs of 600 frames each) was randomly selected to obtain a training-set (Fig. 1, step C.1 and Table 2). The trainingset comprised three different muscles (dorsal interossei, gastrocnemius, rectus femoris) that had a Heckmatt-score of either 1 or 2 . One clinical observer (NvA) marked the time frames in which fasciculations occurred in this training set by using a frame-by-frame visual analysis (Fig. 1, step C.2). This resulted in labeled example data, which we used to develop and optimize our detection algorithm for the detection of fasciculations (see section D and Fig. 1, step D). These initially found fasciculations were later compared to the number of fasciculations found with the help of the developed algorithm to evaluate its performance.

\section{D. The algorithm for automatic detection of movement}

Our motion detection method (implemented in Matlab, MathWorks Inc., Natick, MA, USA) comprised a modified background 
Table 1

All patient data.

\begin{tabular}{|c|c|c|c|c|}
\hline Participant & Sex & Age at exam & Diagnosis & Examined muscles \\
\hline \#1 & Male & 62 & Amyotrophic lateral sclerosis & $\begin{array}{l}\text { biceps brachii } \mathrm{L}^{\mathrm{T} 4} \\
\text { Rectus femoris } \mathrm{R} \\
\text { Masseter } \mathrm{R} \\
\text { Flexor carpi radialis } \mathrm{R} \\
\text { First dorsal interosseous } \mathrm{L}\end{array}$ \\
\hline \#2 & Male & 74 & Amyotrophic lateral sclerosis & Rectus femoris $\mathrm{R}, \mathrm{L}$ \\
\hline \#3 & Male & 64 & Amyotrophic lateral sclerosis & $\begin{array}{l}\text { Medial gastrocnemius } \mathrm{L} \\
\text { Tibialis anterior } \mathrm{R}^{\mathrm{T} 1} \\
\text { First dorsal interosseous } \mathrm{L}\end{array}$ \\
\hline \#4 & Female & 12 & Focal inflammatory neuropathy & $\begin{array}{l}\text { Flexor carpi radialis } \mathrm{R} \\
\text { Medial gastrocnemius R,L }\end{array}$ \\
\hline \#5 & Female & 53 & Lumbosacral radiculopathy & $\begin{array}{l}\text { Masseter R } \\
\text { Rectus femoris L } \\
\text { Medial gastrocnemius } \mathrm{L}^{\mathrm{TR} 4} \\
\end{array}$ \\
\hline \#6 & Male & 54 & Rigid spine myopahty & Medial gastrocnemius $\mathrm{L}^{\mathrm{TR} 3}$ \\
\hline \#7 & Female & 9 & Hereditary motor and sensory neuropathy type $4 c$ & $\begin{array}{l}\text { Medial gastrocnemius } \mathrm{L}^{\mathrm{T} 3} \\
\text { Tibialis anterior } \mathrm{L}\end{array}$ \\
\hline \#8 & Male & 64 & Progressive spinal muscular atrophy & $\begin{array}{l}\text { Flexor carpi radialis } \mathrm{R} \\
\text { First dorsal interosseous } \mathrm{L}^{\mathrm{TR} 2} \\
\text { Medial gastrocnemius } \mathrm{R}, \mathrm{L} \\
\underline{\text { Rectus femoris } \mathrm{R}^{\mathrm{T} 5}}\end{array}$ \\
\hline \#9 & Male & 58 & Cramp fasciculation syndrome with S1 radiculopathy & First dorsal interosseous $\mathrm{R}, \mathrm{L}^{\mathrm{TR} 1}$ \\
\hline \#10 & Male & 41 & Myalgia and exercise intolerance, no underlying neuromuscular disorder found & $\begin{array}{l}\text { Medial gastrocnemius } \mathrm{R} \\
\text { Tibialis anterior } \mathrm{L} \\
\text { Flexor carpi radialis } \mathrm{L}\end{array}$ \\
\hline \#11 & Male & 47 & Reinnervated muscle, no underlying neuromuscular disorder found & $\begin{array}{l}\text { Geniohyoid } \\
\text { Masseter R }\end{array}$ \\
\hline & & & & $\begin{array}{l}\text { Flexor carpi radialis } \mathrm{R}^{\mathrm{T} 2} \\
\text { Rectus femoris } \mathrm{R}, \mathrm{L}^{\mathrm{TR} 5} \\
\text { Tibialis anterior } \mathrm{L} \\
\text { Sternocleidomastoid } \mathrm{R} \\
\text { medial gastrocnemius } \mathrm{R}, \mathrm{L}\end{array}$ \\
\hline
\end{tabular}

$\underline{\mathrm{TR}}=$ training-set, $\underline{\mathrm{T}}=$ Test-set.

Table 2

Training-set patient data.

\begin{tabular}{llll}
\hline Participant & Muscle & Heckmatt-score & $\begin{array}{l}\text { Initial } \\
\text { number of } \\
\text { fasciculations }\end{array}$ \\
\hline Training \#1 & First dorsal interosseous L & 1 & 6 \\
Training \#2 & First dorsal interosseous L & 1 & 3 \\
Training \#3 & Medial gastrocnemius L & 2 & 13 \\
Training \#4 & Medial gastrocnemius L & 1 & 3 \\
Training \#5 & rectus femoris L & 1 & 3 \\
\hline
\end{tabular}

Table 3

Test-set patient data.

\begin{tabular}{llll}
\hline Participant & Muscle & Heckmatt-score & $\begin{array}{l}\text { Initial number of } \\
\text { fasciculations }\end{array}$ \\
\hline Test \#1 & Tibialis anterior R & 3 & 4 \\
Test \#2 & Flexor carpi radialis R & 1 & 0 \\
Test \#3 & Medial gastrocnemius L & 3 & 1 \\
Test \#4 & Biceps brachii L & 2 & 0 \\
Test \#5 & Rectus femoris R & 1 & 1 \\
\hline
\end{tabular}

subtraction (or frame-difference) method, which is a frequently used step in many optical motion capturing systems (Ramya and Rajeswari, 2016). By subtracting static background from an image sequence, static areas will cancel out to zero revealing the nonzero areas where motion has occurred. In ultrasound applications this method has for example been applied to remove vascular wall tissue movements from image sequences, which enhances the remaining signal of blood in the ultrasound images for blood flow estimation (Jin and Wang, 2007).
To detect movement, we subtracted the background image from the contiguous image sequence. The background image at every time point $t$ was computed by averaging a series of images (double sided window size $=31$ frames). Subsequently, the difference image was divided by the standard deviation of the image within the same time series. This results in a measure that yields the extent of pixel intensity variability (PIZ-score, see Eq. (1)) which is the equivalent of the z-score of pixel intensity and represents tissue motion (see also Figs. 2 and 3).

$\operatorname{PIZ}(m, n, t)=\frac{|f(m, n, t)-\bar{f}(m, n, t)|}{s(m, n, t)}$

where $m$ and $n$ are the pixels indices, and $t$ the time frame.

To cluster pixels into event regions, e.g., regions of the ultrasound image with suspected motion, the PIZ values were 2D Gaussian smoothed ( $\sigma=10$ pixels) and connected in space and time using 3D flood filling with an empirical derived PIZ score of 1.5. Pixels without noteworthy signal (i.e., $\bar{f}(m, n, t)<30)$ or that have a low variation ( $($ i.e., $s(m, n, t)<5)$ were ignored. The maximum of the $P I Z$ for every time point converts the $2 \mathrm{D}+\mathrm{t}$ image information into a 1-D signal. The magnitude of that signal represents the likelihood of an ultrasound frame containing motion. Based on the first analysis of the training-set an arbitrary threshold PIZ of 1.5 was chosen to include all fasciculations identified by the clinical observers (see Fig. 1, step D and Fig. 4) and to establish future detection of clinical evident fasciculations by the algorithm.

\section{E. Computer-aided detection of fasciculations}

The algorithm developed in step D was next tested on the training-set, to assess whether the clinician had missed any fasciculations in the manual annotation (see Fig. 1E.1), and on five addi- 


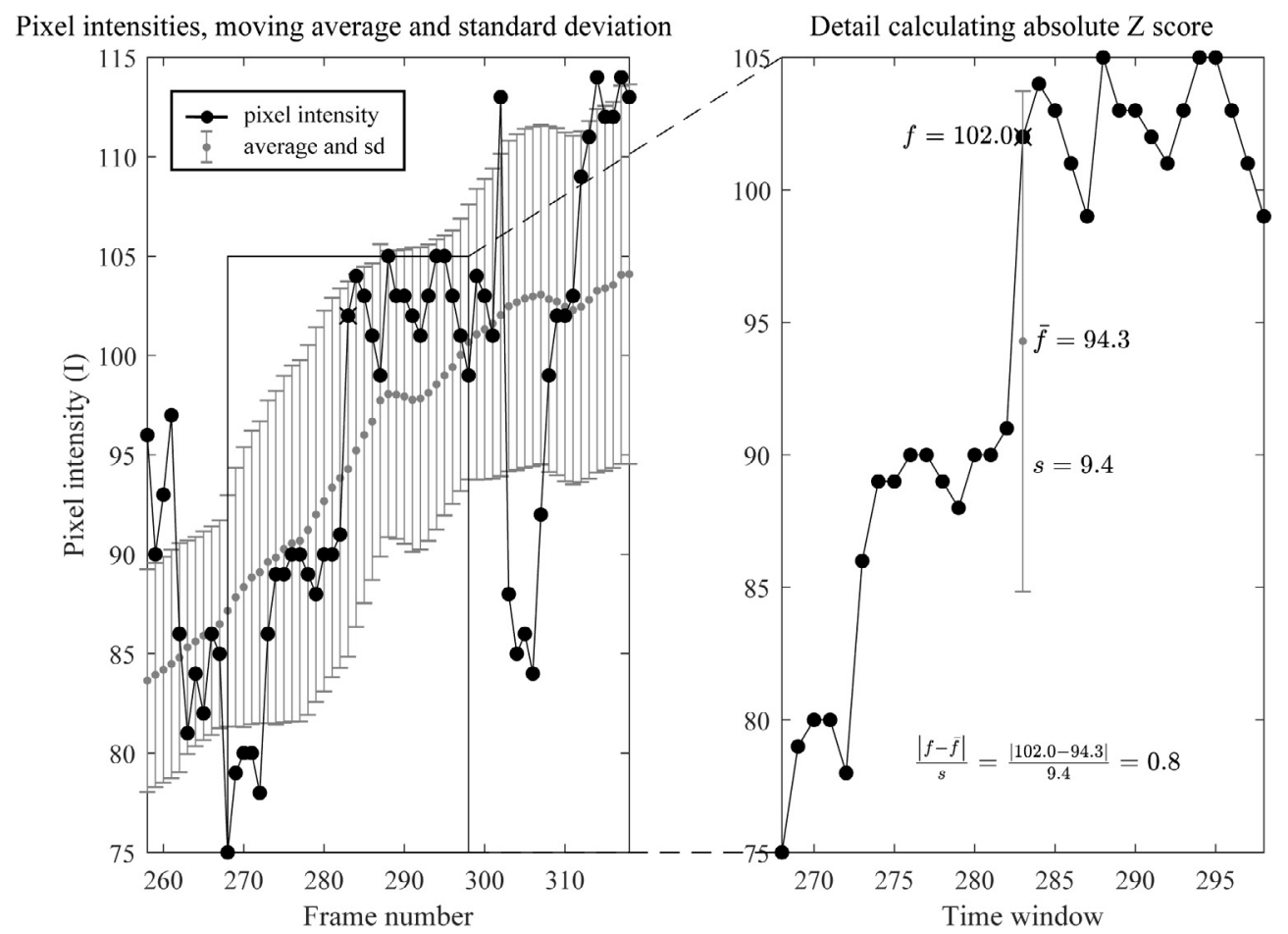

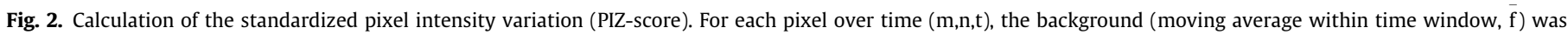
subtracted from the intensity (f) value and divided by the standard deviations.

tional ultrasound sequences (the test-set) to evaluate the performance of the algorithm on unfamiliar data i.e., data not used to 'train' the algorithm (see also Fig. 1E.2). The test-set comprised five muscles (biceps brachii, flexor carpi radialis, rectus femoris, tibialis anterior, gastrocnemius) with Heckmatt-scores between 1 and 3 (see Table 3). The test-set contained manually selected ultrasound sequences with low fasciculations severity scores, and also included videos without the presence of fasciculations, which allowed us to test if our algorithm did not miss any 'critical' cases i.e., the absence of fasciculations that would alter a diagnosis.

The outcome of the algorithm consisted primarily of a number of movement events for each ultrasound video. Every image was next divided into 25 areas, numbered as seen in Fig. 5 to further guide the clinical observers. For every movement event, the corresponding time frame and location within the ultrasound image were recorded, and presented to the clinical observer, who then specifically evaluated these frames in the video to classify the events into the following categories: fasciculations, voluntary contractions, contraction pseudotremor (i.e., slight antigravity contraction of one or a few enlarged motor units), vascular pulsations, image artefacts, or any other movement type. The results of the algorithm-guided clinical annotation were compared to the initial visual annotation by the clinicians (see Fig. 1, step E) to detect any differences.

\section{F. Physiologic parameters}

Besides fasciculations, the ultrasound videos also contained other movement types, as expected. To enable automatic classification of these movements and separate them from the fasciculation, the following five parameters were calculated for every detected movement event in the training-set:

1. Duration (\# frames): The number of ultrasound frames that correspond to a detected event.

2. Area (\# pixels, $\mathrm{mm}^{2}$ ): The number of pixels that are involved in a detected event and the area they cover.
3. Occurrence: The average of instances the pixels within the event are involved in the total number of detected events.

4. Periodicity $\left(I^{2}\right)$ : For every pixel within the event we compute the power spectrum of the PIZ signal. The average of the maxima of the power spectra represents the periodicity. We searched for the maximum within the expected normal range of frequencies for the heart rate (50-120 bpm).

5. Concurrent global deviation (I): The average of $s(m, n, t)$ for all pixels of the entire image frame corresponding to the detected event. This measure represents the amount of surrounding motion.

\section{Results}

In this section, we first present the outcomes of the manually and guided annotation of fasciculations in the training-set (Fig. 1, step E.1) and subsequently test-set of ultrasound image sequences (Fig. 1, step E.2). Secondly, we present the physiologic features we calculated for every motion event (Fig. 1, step F).

\subsection{Manual versus computer algorithm-guided fasciculation detection}

Table 4 summarizes the outcomes of the manually and algorithm-guided detection of fasciculations. The initial manually annotated number of fasciculations (NF) in the training-set ranged between $\mathrm{NF}=1$ and $\mathrm{NF}=6$ within an ultrasound image sequence, with a total of $\mathrm{NF}=19$ for all videos. When the observer subsequently reviewed the ultrasound sequences again with guidance of the movement events listed by our algorithm, more fasciculations were found, with a total NF of 49. The total difference between manually and algorithm-guided annotation within the training-set was 30 , and ranged between 0 and 14 per video of the training-set. Fig. 6 shows that all manual annotated fasciculations were found, except for one fasciculation event that was annotated as a contraction pseudotremor in the algorithm-guided evaluation. 
A.
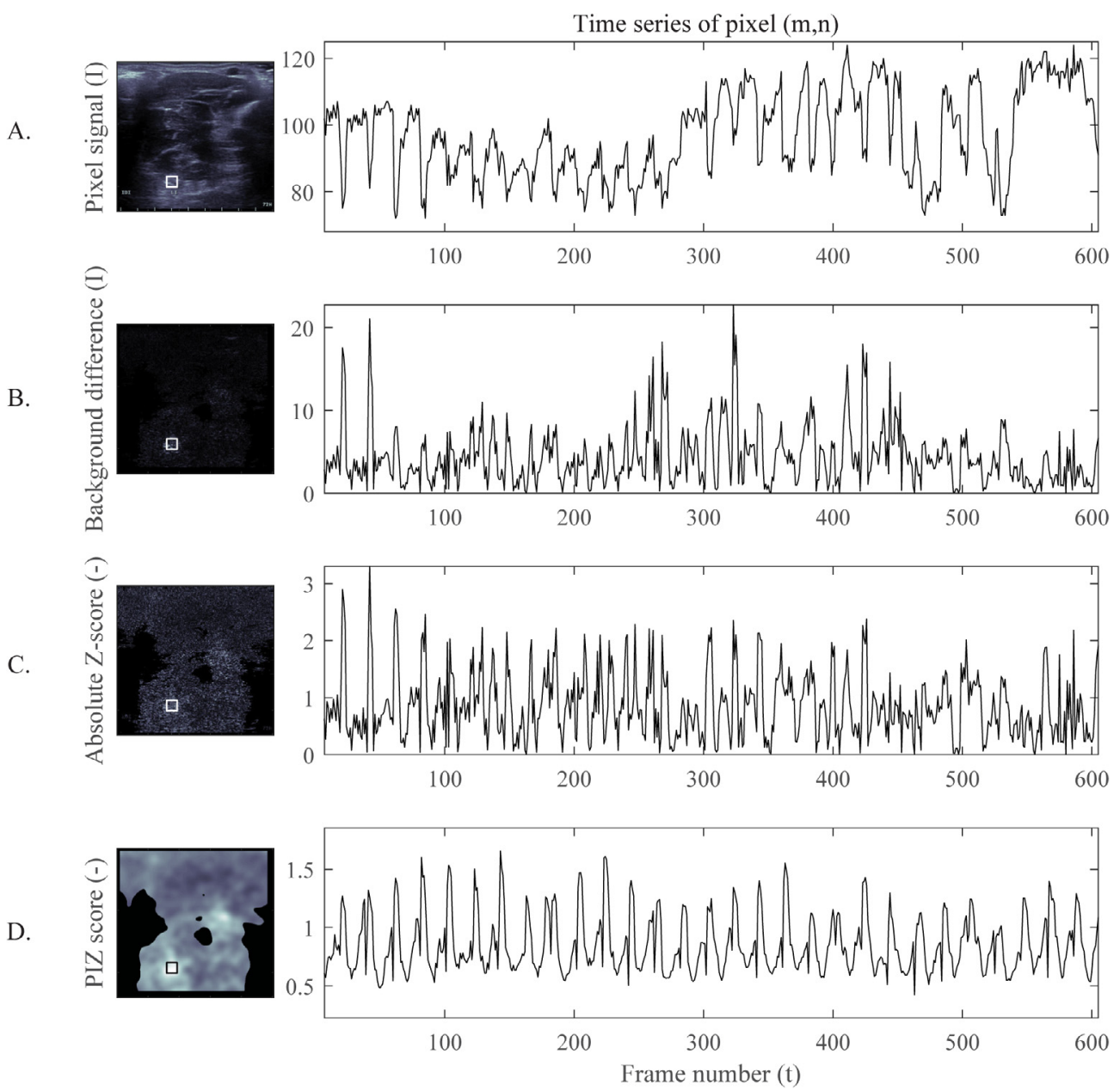

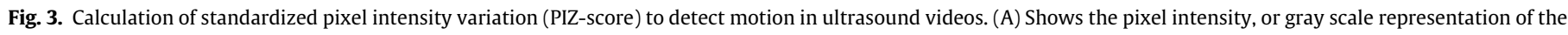

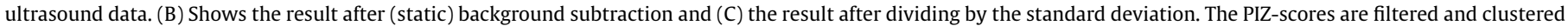
resulting in (D). Please note that the pixel $(\mathrm{m}, \mathrm{n})$ was selected inside a region where vascular pulsation was visible resulting in the periodic PIZ-score signal.

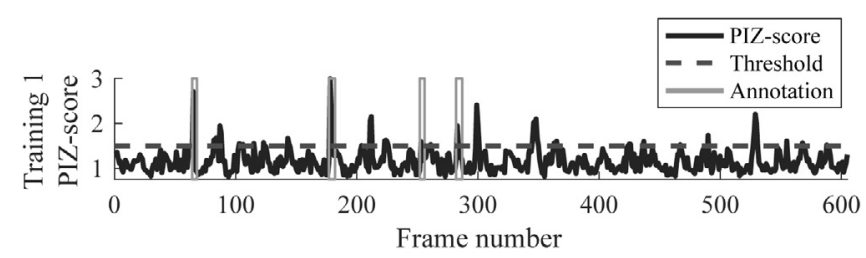

Fig. 4. Example of manually annotation of fasciculations for an ultrasound sequence from the training-set and the derivation of the pixel intensity variation (PIZ-score). The black line represents the maximum PIZ-score of the entire image over time, a threshold of 1.5 was chosen to include all 4 manually annotated fasciculations (gray vertical lines). When the PIZ-score value exceeds the threshold, the motion was classified as a movement event.

Of note, the number of manual (frame-by-frame) annotated fasciculations in training video \#3 had a very wide range between the observers from the initial visual screening: from 13 to 1 . These movements were classified as contraction pseudotremor in the frame-by-frame annotation. With the aid of the computer algorithm the number of detected fasciculations again increased, to 15. This indicates that there is a considerable effect of observervariability possibly related to the presence of contraction pseudotremor.

From the original 600 frames per video in the training-set, only a fraction of the data (145 movement events from 3000 frames, or $\sim 5 \%$ ) needed to be reviewed to classify the automatically detected motion events, depending on the number of detected events, which were 29 on average and ranged between 20 and 39 per video for the fasciculations combined with all other movements. Especially contractions, both voluntary but also contraction pseudotremor, were observed in almost every recording, and resulted in additional detected events besides fasciculations.

For the ultrasound image sequences from the test-set, we observed a global decrease in annotated fasciculations with the help of the computer algorithm (see also Table 4). Initially the observers annotated a total of $\mathrm{NF}=6$ in all videos of this test-set, whereas with the computer guidance fewer fasciculations were annotated $\mathrm{NF}=4$. The difference between manually (initial scoring) and computer guided annotation within this test-set was within the range of: -3 and 1 . We observed that the decrease of total number of fasciculations found was the result of a single video (test \#1), in which some of the fasciculations occurred in the same region as a contraction pseudotremor and were not detected as a result of large pixel variation over time in that area (i.e., a high standard deviation in the image sequence results in low PIZ-score). The number of fasciculations in the other videos were correctly found or increased similar to the results of the training-set. The total number of automatically detected movement events was 44 (out of 3000 frames, or 1.5\%) and showed that there were less movements in the videos within the test-set compared to the training-set. On average the videos in the test-set contained 9 movements, with the number of movement events 


\begin{tabular}{|c|c|c|c|}
\hline Event & Time frame & Location & Area \\
\hline 1 & 32 & C3 & 1342 \\
\hline 2 & 105 & B3 & 18074 \\
\hline 3 & 162 & B2 & 7296 \\
\hline 4 & 168 & D3 & 7675 \\
\hline 5 & 188 & B2 & 332 \\
\hline 6 & 222 & B3 & 6343 \\
\hline $\begin{array}{l}\text { mnumber of } \\
\text { detected } \\
\text { movements }\end{array}$ & & & \\
\hline
\end{tabular}

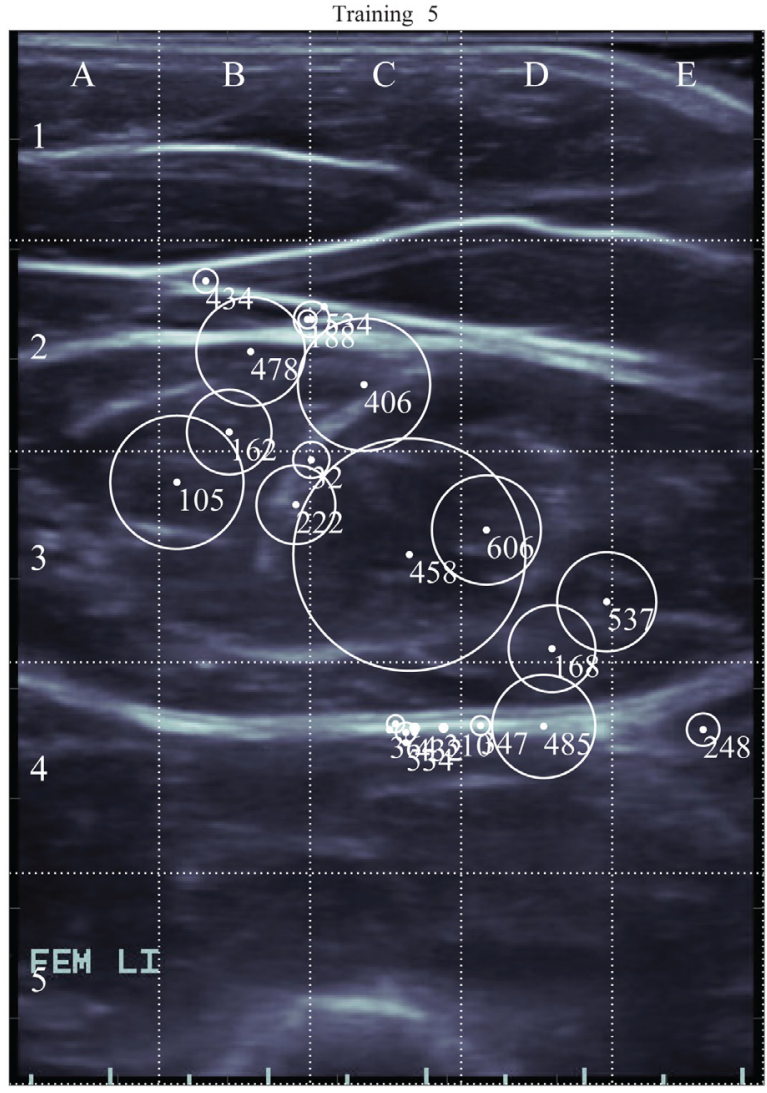

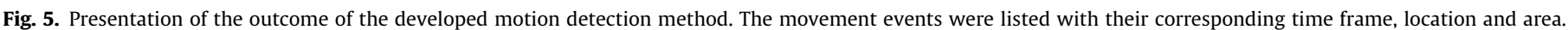

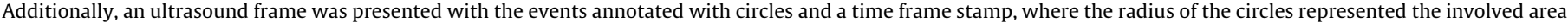
of the event.

Table 4

Manual and computer guided annotations of fasciculations for the individual recordings in the training and test-set and in bold the total number of annotations.

\begin{tabular}{|c|c|c|c|c|c|}
\hline Data & $\begin{array}{l}\text { Initial manual } \\
\text { count }\end{array}$ & $\begin{array}{l}\text { Frame by frame } \\
\text { count } \\
\text { of Fasciculations }\end{array}$ & $\begin{array}{l}\text { Computer guided } \\
\text { count of } \\
\text { Fasciculations }\end{array}$ & $\begin{array}{l}\text { Total automatically } \\
\text { detected } \\
\text { events/movement }\end{array}$ & Other motion observed \\
\hline Training \#1 & 6 & 4 & 8 & 29 & Vascular pulsation, contractions, contractions pseudotremor \\
\hline Training \#2 & 3 & 2 & 5 & 39 & Contractions, contractions pseudotremor, probe motion \\
\hline Training \#3 & 13 & 1 & 15 & 25 & Probe motion, contractions, pseudotremor \\
\hline Training \#4 & 3 & 6 & 15 & 32 & Probe motion, vascular pulsation, contractions pseudotremor \\
\hline Training \#5 & 3 & 6 & 6 & 20 & probe motion, vascular pulsation \\
\hline Training-set (total) & 28 & 19 & 49 & 145 & \\
\hline Test \#1 & 4 & - & 1 & 9 & Contractions pseudotremor, vascular pulsation \\
\hline Test \#2 & 0 & - & 0 & 11 & Probe motion, vascular pulsation \\
\hline Test \#3 & 1 & - & 1 & 8 & Contractions \\
\hline Test \#4 & 0 & - & 0 & 5 & Contractions, probe motion \\
\hline Test \#5 & 1 & - & 2 & 11 & Vascular pulsations, probe motion \\
\hline Test-set (total) & 6 & - & 4 & 44 & \\
\hline
\end{tabular}

Please note that the values presented in the second column are the same as presented in Tables 2 and 3.

ranging between 5 and 11 . Consequently, clinicians had to review fewer frames in these videos compared to the videos in the training-set.

\subsection{Physiologic parameters of detected movements}

Fig. 7 illustrates the average values of the parameters of duration, area, occurrence, periodicity and surrounding motion for every classified movement event from the training-set (see also Fig. 1, step F). Duration and area were not found to distinguish between fasciculations and other movement events. Occurrence reflects the repetitive behavior of events and was highest for contractions pseudotremor with an average value of $11.4 \pm 3.7$ times.
In other words, pixels within the region of a pseudotremor contraction were involved in 11.4 additional detected movement events in the video (most likely another pseudotremor contraction). Vascular pulsations were clearly recognizable with the use of the periodicity parameter, which had the highest average value of $5.9 \pm 5.2$ (power, $I^{2}$ ). Motions that were classified as artefacts, were frequently caused by probe motion, and these events showed the largest pixel intensity variation for the entire image $6.9 \pm 6.3(I)$.

\section{Discussion}

In this work we developed the first steps of an algorithm for the automatic detection and specification of motion in muscle ultra- 


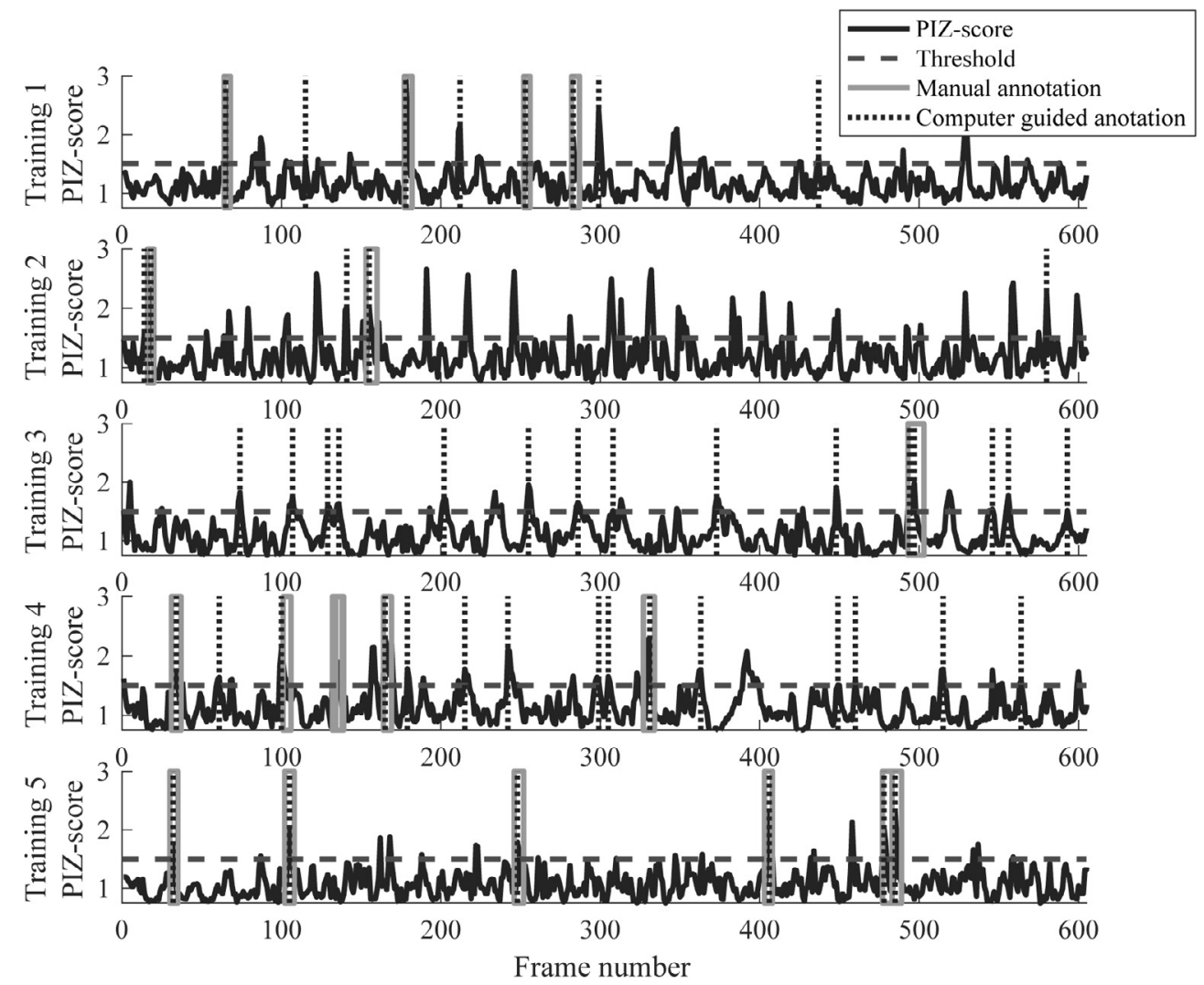

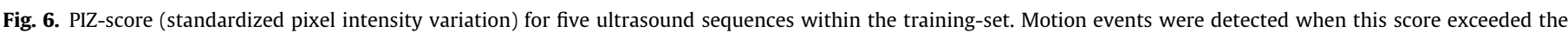

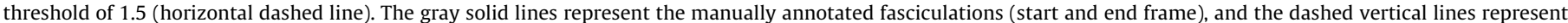

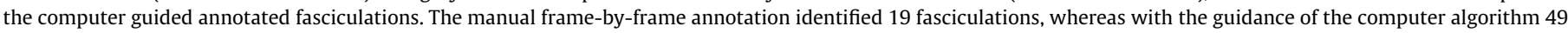
fasciculations were found.
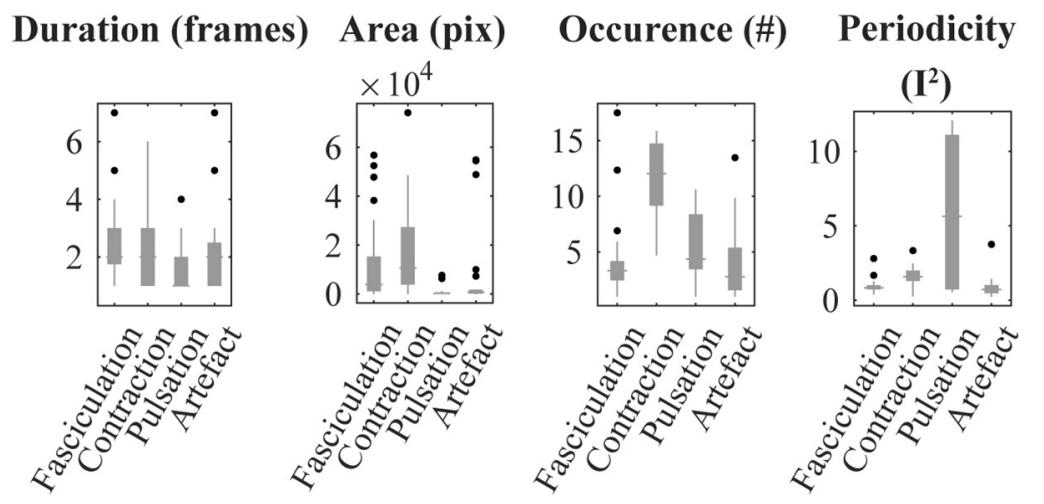

\section{Concurrent}
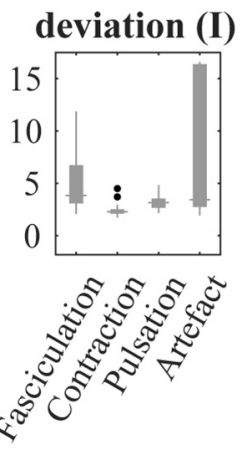

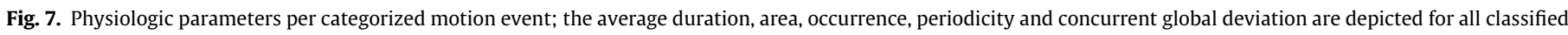
events in the training-set.

sound image sequences acquired during clinical practice. We found that algorithm found all manual detected fasciculations in the testset, except for three fasciculations in one challenging video, indicating a high level of accuracy; 4/5 videos in the-test were correctly annotated with the help of the algorithm. This makes an automated approach an attractive method to objectively screen for the presence of fasciculations in muscle ultrasound videos, and confirms the findings of a previous study by Harding et al. (Harding et al., 2016).

Using the algorithm, clinicians were able to detect more fasciculations than using offline visual analysis alone, and the use of the algorithm to prescreen the videos could save clinicians time that would have been needed to review the full ultrasound video. There were in total 189 movement events found by the algorithm in the training and test-set combined (6000 frames total), depending on the number of frames required to investigate a movement event, this will save considerable time. For instance, when 10 frames are required to investigate a movement event this will lead to a time reduction of approximately $70 \%$ ( 189 events * 10 frames/6000 frames). This shows that computer-guidance may also be an attractive approach for fasciculation detection that will help improve sensitivity and saves a considerable amount of time for the human observer.

Furthermore, with help of the algorithm combined with revision by the clinician, the ultrasound videos consistently were found to contain additional movements, especially repetitive con- 


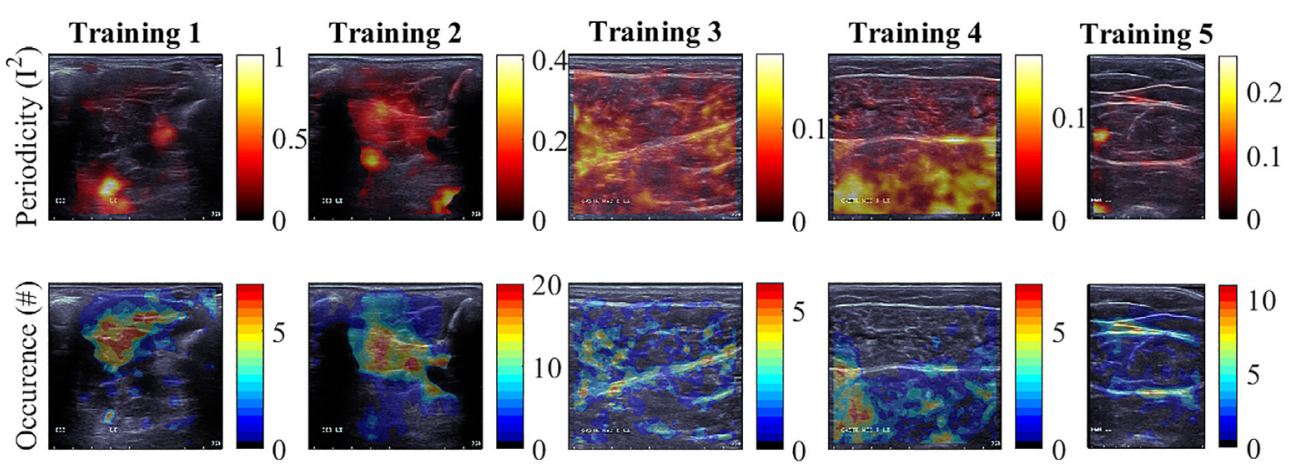

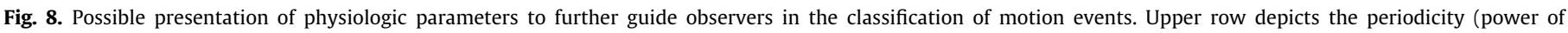

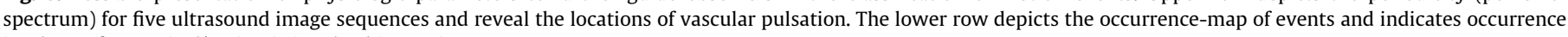
i.e., how often a pixel/region is involved in motion events.

tractions of single motor units (either voluntary or anti-gravity contraction). These movements can potentially confound the visual analysis of muscle ultrasound videos, especially when it is performed online. The movements were found to have specific characteristics that would tell them apart from each other. Using the computer algorithm enhanced with a standard detection method for these features is expected to give good detection accuracy and prevent confounding of the different movements, thus improving specificity of the muscle ultrasound video analysis.

The analysis of the test-set indicate that the algorithm is capable of coping with a large variability of ultrasound data that exist in clinical examinations. It included acquisitions from different muscles of patients with varying diseases and disease stages. Although there was a good agreement between the number of fasciculations found, it is unclear if the automatically detected events included the originally annotated fasciculations, since we only have the number of fasciculations and not the starting frames within the test-set. The algorithm failed to present all fasciculations to the observer in one of the test-set recordings (test\#1). A thorough inspection of this data revealed that the missed fasciculations occurred at the location of a pseudotremor, which confounds the automated detection by presenting a high level of background movement in a certain image area. Further research is required to optimize the algorithm for this sort of challenging data. Further, certain confounding movements, such as contraction pseudotremor or anti-gravity contractions can be prevented by making sure the patient is completely relaxed during the examination.

Agreement between observers has been shown to be influenced by the number of fasciculations present in the data being analyzed; fewer fasciculations corresponds to lower agreement (Harding et al., 2016). Consequently, a comprehensive evaluation of the algorithm on the test-test is difficult since the initial annotations were scored by different observers. Additional studies on the reliability of the algorithm should include a more comprehensive collection of ground-truth data, such as the collection of EMG data and data on intra/inter-observer variability.

The additional physiologic data extracted from the ultrasound movement events in this study indicate these parameters can be used to discriminate fasciculations from other types of motion. Vascular pulsation events showed highest periodicity values and can be excluded using this information. Additionally, probe motion artefacts might be discarded using the average pixel variation of the entire image. To improve the computer-aided detection, the physiologic data can be displayed as an overlay over the ultrasound images to help the observer in the classification (see Fig. 8). Ideally, these parameters are incorporated in a machine learning algorithm, such as a random-forest classifier or neural network, for fully automatic detection and classification. The feasibility of using machine learning for this application will be studied in future work. This requires further investigation of the variability of the derived parameters/features. For example, the magnitude of the periodicity will be affected negatively by probe motion. Therefore, it might be important to have more strict requirements of ultrasound recordings and acquisition protocols to prevent unnecessary difficulties in the image processing. Furthermore, the method could be optimized for each specific muscle, since it might be the case that other parameters are optimal for different muscles.

The method proposed for automated fasciculation detection is a computationally cheap (i.e., fast) alternative for other motion detection techniques such as optical flow (Harding et al., 2016). However, the proposed method is not capable of quantifying motion. Additional information, such as the characterization of the motion pattern may help distinguish between fasciculations and other involuntary contractions. For example, myokymia appears as "brief but sustained, tractive movements" of the muscle, which contrasts with the brief rotary muscle movements that are typical for fasciculations (Simon, 2015). Our method may be used as a pre-processing step to detect ultrasound frames that contain motion, and subsequently, extract quantitative information of the tissue motion using optical flow or speckle tracking (Gijsbertse et al., 2017a, 2017b). Further exploration of the use of the proposed algorithm will include its potential for detecting other biomarkers in neuromuscular diseases, such as myokymia or fibrillations.

In conclusion, the findings above confirm the potential clinical usefulness of an automated approach to movement analysis in muscle ultrasound videos. The derived additional physiologic features together with quantitative techniques have the potential to improve diagnosis and may lead to fully automatic classification of motions in neuromuscular diseases.

\section{Acknowledgements}

The research leading to these results has received funding from the European Research Council under the European Union's Seventh Framework Programme (FP/2007-2013)/ERC Grant Agreement n. 323091 awarded to N. Verdonschot.

\section{Conflict of interest statement}

None of the authors have potential conflicts of interest to be disclosed.

\section{References}

Geevasinga N, Loy CT, Menon P, de Carvalho M, Swash M, Schrooten M, et al. Awaji criteria improves the diagnostic sensitivity in amyotrophic lateral sclerosis: A 
systematic review using individual patient data. Clin Neurophysiol 2016;127:2684-91.

Gijsbertse K, Goselink R, Lassche S, Nillesen M, Sprengers A, Verdonschot N, et al. Ultrasound imaging of muscle contraction of the tibialis anterior in patients with facioscapulohumeral dystrophy. Ultrasound Med Biol 2017a;43:2537-45.

Gijsbertse K, Sprengers AM, Nillesen MM, Hansen HH, Lopata RG, Verdonschot N, et al. Three-dimensional ultrasound strain imaging of skeletal muscles. Phys Med Biol 2017b;62:596-611.

Grimm A, Prell T, Decard BF, Schumacher U, Witte OW, Axer H, et al. Muscle ultrasonography as an additional diagnostic tool for the diagnosis of amyotrophic lateral sclerosis. Clin Neurophysiol 2015;126:820-7.

Harding PJ, Loram ID, Combes N, Hodson-Tole EF. Ultrasound-based detection of fasciculations in healthy and diseased muscles. IEEE Trans Biomed Eng 2016;63:512-8.

Heckmatt JZ, Leeman S, Dubowitz V. Ultrasound imaging in the diagnosis of muscle disease. J Pediatr 1982;101:656-60.

Jin D, Wang Y. Doppler ultrasound wall removal based on the spatial correlation of wavelet coefficients. Med Biol Eng Comput 2007;45:1105-11.
Misawa S, Noto Y, Shibuya K, Isose S, Sekiguchi Y, Nasu S, et al. Ultrasonographic detection of fasciculations markedly increases diagnostic sensitivity of ALS. Neurology 2011;77:1532-7.

Pillen S, Nienhuis M, van Dijk JP, Arts IM, van Alfen N, et al. Muscles alive: ultrasound detects fibrillations. Clin Neurophysiol 2009;120:932-6.

Ramya P, Rajeswari R. A modified frame difference method using correlation coefficient for background subtraction. Procedia Comput Sci 2016;93:478-85.

Regensburger M, Tenner F, Mobius C, Schramm A. Detection radius of EMG fo fasciculations: empiric study combining ultrasonography and electromyography. Clin Neurophysiol 2017;129:487-93.

Reimers CD, Ziemann U, Scheel A, Rieckmann P, Kunkel M, Kurth C. Fasciculations: clinical, electromyographic, and ultrasonographic assessment. J Neurol 1996;243:579-84.

Scholten RR, Pillen S, Verrips A, Zwarts MJ. Quantitative ultrasionography of skeleta muscles in children: normal values. Muscle Nerve 2003;27:693-8.

Simon NG. Dynamic muscle ultrasound - another extension of the clinical examination. Clin Neurophysiol 2015;126:1466-7. 